\title{
Automatic, Body Measurements Based Generation of Individual Avatars Using Highly Adjustable Linear Transformation
}

\author{
Andreas Volz, Rainer Blum, Sascha Häberling, and Karim Khakzar \\ Fulda University of Applied Sciences, Department of Applied Computer Sciences, \\ Marquardstr. 35, 36039 Fulda, Germany \\ Andreas.Volz@tux-style.de, \\ \{Rainer.Blum, Sascha.Haeberling, \\ Karim.Khakzar\}@informatik.hs-fulda.de
}

\begin{abstract}
In this paper part of the work of the research project 'IntExMa' is presented, that aims at the development of an interactive, in-store sales support system focusing on the virtual 'try-on' of clothing. Customers are provided with highly realistic, individualized, three-dimensional virtual counterparts, derived from a small number of body measurements. The paper describes in detail the approach followed for the required automatic generation of avatars. It is based on a sophistication of the well-known concept of parametric deformation. The solution presented here is characterized by a range of means that provide highly flexible, fine-grained adjustments of the linear transformation process leading to precisely controllable results.
\end{abstract}

Keywords: body measurements, parametric deformation, individualized avatars.

\section{Introduction}

\subsection{The IntExMa Project}

The goal of the IntExMa project (Interactive Expert System for Made-to-measure Clothing) is to provide an in-store installation for small and medium sized businesses, which provides a virtual shopping experience to the customers. The special context addressed by the project at the moment are made-to-measure shirt shops. Customers of these shops cannot see the final product in real life, as this is specifically assembled and tailored according to their specifications. Although examples of fabrics and other components are available in the store, the final assembly is not visible to the customers before delivery.

This is where the IntExMa system offers added value by providing a "virtual tryon" solution. It consists of a virtual representation of the customer (avatar) on a large display screen wearing the not yet produced shirt. The latter can be change according to the product range offered in the shop. The setup's intention is to create the impression of a "virtual mirror". This way, customers shall get a better understanding of the 
look and fit of the final product, leading to well-founded buying decisions. In order to reach this goal, an advanced cloth simulation is used to render the virtual shirts close to reality.

\subsection{Objectives}

User reactions have shown, that the overall acceptance of the IntExMa system by customers does not only depend on the quality of the displayed clothes but also on the appearance of the avatar wearing the virtual shirt. Giving the users the impression to look at their virtual counterparts seems to be an important aspect. To offer a limited selection of avatar shapes has proven not to be sufficient. Avatars are required that are an individual representation of the customers, at least to the required extent for personal identification.

A crucial point is the rather rare data that is available about the clients. The basic concept of IntExMa is to provide an affordable system solution which can seamlessly be integrated into existing point of sale activities and environments. A high-end approach for customer digitization with the help of body scanners or the like is not feasible in this context of use. Therefore, it was decided to design, implement and evaluate an avatar generation system that works with the limited set of body measurements anyway taken from the customer by the sales person by hand. In case of a shirt these are typically less than ten different measures.

This paper presents a method to solve this problem. It describes an approach to create a three-dimensional avatar, based on a given, limited set of measures taken from a real-life person. This method is based on a sophistication of the concept of "parametric deformation" or "keyframe morphing" [1,2], which is, for example, implemented in the well-known 3D figure design application Poser ${ }^{\circledR}$.

Keyframe morphing is a method to change the shape of a given three-dimensional object by re-locating its vertices. Vectors for those vertices are defined to describe a linear deformation path. A group of those vectors is called a morph target, which usually covers only a subset of vertices. A scalar defines the impact of this vector and, thus, the dimension of this re-location.

To fulfill the described requirements of the IntExMa system, additional functionality has to be provided: Avatars that match the entered measures closely shall be produced automatically. No other currently available system known to the authors has this required characteristic.

Releasing the results of this work as open source makes them available to the public in order to be used on products with similar demands. A project that could already profit is the MakeHuman Project [3], which is specialized on creating highly realistic three dimensional humanoids (cf. Section 3.4).

\section{Deformation Process}

In order to transform the base avatar into a model that complies with the desired body measurements, a morphing algorithm is introduced. It uses a measuring algorithm to obtain information about pre-defined measuring sections of the body. These are used to evaluate the correctness of the result, by comparing these values to the reference 
values. Those may include circumferential as well as range measures, to be able to set the girth of certain body parts as well as their length.

Morph targets are used to define vectors for the deformation of a defined set of vertices from the complete model. At least one morph target is assigned to every measuring section in order to be able to achieve the final deformation value independently from the rest of the measuring sections.

The general process consists of discrete alternating steps of measuring and deformation and ends by reaching the pre-defined values with the given tolerance.

\subsection{Deformation Calculation}

The deformation procedure shown in this paper is an expert system that makes use of linear transformation of vertices. As a base for this transformation a threedimensional human model is used. The algorithm itself is independent of the amount of vertices in the model, but it is crucial that the topology of vertices in the base model corresponds to the one of the morph target - although the morph target can and usually does cover a subset of the base model's set of vertices. This way many overlapping and non-overlapping targets can be defined for a base model and applied concurrently, either fully or partly.

The deformation vector is computed by subtracting the vertice in the base model from the associated vertice within the morph target. A scalar $m$ is influencing the application of a target on the deformation. Thus, only the length of the deformation vector is manipulated. A value of $m=1$ doesn't change the length at all. For $0<m<1$ the impact of the vector is decreased, resulting in a partial deformation. Negative values of $m$ lead to a reversion of the deformation vector. Usually, applying a reversed deformation leads to unsatisfying results and can only be used in rare cases, recommending only the interval $(0,1)$ for predictable results.

\subsection{Measurement}

Since multiple targets allow for independent deformation of the body parts, a way to measure these deformation results at various positions within the model independently had to be found. Therefore, we introduce a technique that measures the avatar with so-called hotspots which can be distributed around the body.

This hotspot technique is similar to the well-known concept of feature points, which, for example, is defined by the H-Anim Standard [4] for humanoids, but offers some necessary extensions. A hotspot contains several feature points in a specified order, thus defining a complex measuring section like the girth of an arm. Figure 2 shows an avatar with some measuring sections highlighted.

The hotspot concept is an easy and efficient way, which allows the calculation of the distances between all the ordered feature points $h s_{i}$ within the hotspot, adding them to the final measure value $l$, as shown in the following equation:

$$
l=\sum_{i=0}^{n-1}\left|\overrightarrow{h s_{i}}-\overrightarrow{h s_{i+1}}\right|
$$




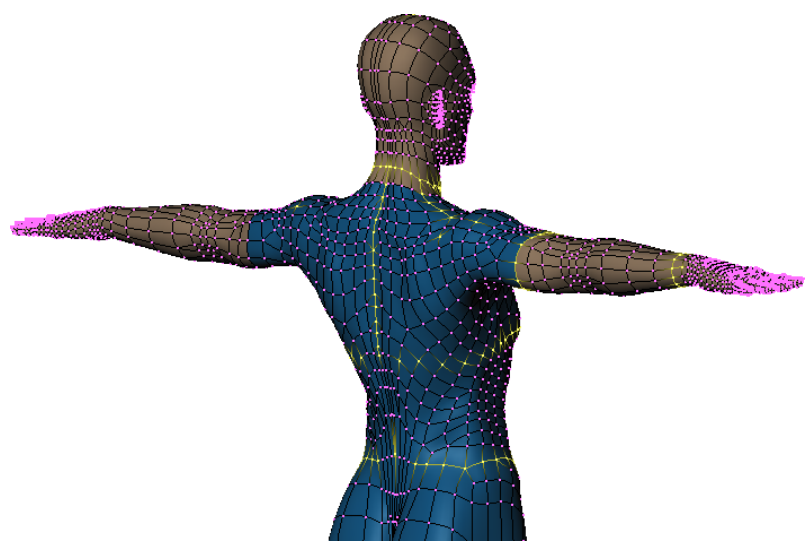

Fig. 1. Avatar with highlighted measuring sections/hotspots

The actual value of the result depends on the scale of the model. Thus, in order to obtain real-world units, the scale of the base model has to be considered.

\subsection{Automatic Deformation}

Of course, deformations could be done directly by setting the scalars of each morph target. But with the given input data at hand, i.e. base model, morph targets and body measures, an automatic process can perform this deformation iteratively to end up with a model that fits the desired input measures.

The principle of deformation described here is independent from material masses and units. By the definition of a suitable scale the associated measure can be computed to each measuring section. A suitable target, at least within the defined range, must have a linear course. This characteristic is used for the determination of the necessary deformations.

As an initializing step, a linear gradient for a defined range is determined using test measurements. Then a first estimation of the necessary morph value can be computed. The deformation is then applied to the avatar and a new test measurement of the appropriate measuring section is taken. If the linear process is sufficiently precise and the delta is within a defined tolerance this iterative deformation process ends. In many cases the linear value gets very close to the final value, but often it's not within the given tolerance. In this case a loop starts which repeats this process of re-measuring and deformation in order to refine the result until the delta is sufficiently small. The computing time rises proportionally with each additional deformation step until the desired accuracy level is reached.

To further control the deformation process, additional configuration input parameters can be provided. As mentioned before, several targets may be applied to the model and overlapping is possible. Furthermore, two overlapping morph targets may cover a number of vertices that are of relevance for one particular measure. For the iterative process to work properly under these circumstances, an order of application must be provided for the targets. 


\subsection{Range Parameterization}

This deformation method is not limited to changing proportions of a base avatar in order to control its body measurements. Its flexibility allows an application for other purposes, like controlling age or gender, as well. In order to achieve this, range parameterization is applied. This technique maps a real world measure onto a scalar for a given target. This way it is possible to define the application of a target like "young" within the range of 6 to 18 "years", resulting in a scalar of $m=1$ for age 6 and $m=0$ for age 18 (regarding a base model representing age 18). Likewise, additional targets could be added to cover other ranges as well, allowing for a fuzzy-like curve.

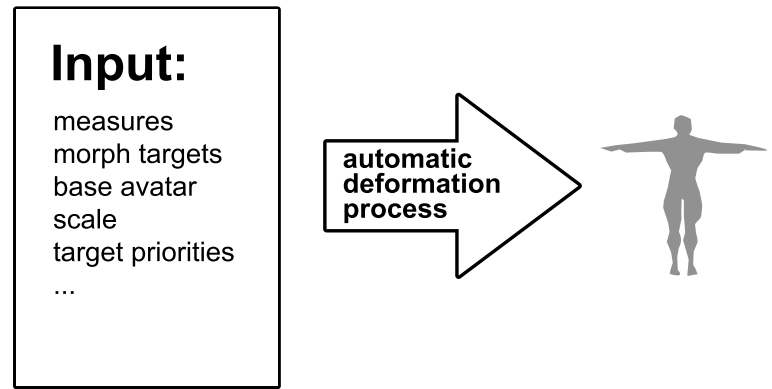

Fig. 2. Principle of the automatic deformation

Fig. 2 depicts the overall process summarizing necessary input elements.

\section{System Design and Implementation}

\subsection{Library Design}

The prototypic implementation of the system is object oriented. The classes were divided into two separated libraries, that can be used independently. The first library was named libanimorph. It implements all low-level tasks like transformation and deformation of the models.

For the automatic deformation process a second library, libavatar, was created on top of libanimorph. It contains the routines that handle the measures in order to perform the automatic deformation process.

Both libraries are released under the GNU Lesser General Public License (LGPL).

\subsection{Content Generation Tools}

In order to support the generation process of the necessary input data, some specialized tools were developed. MakeTarget, a plug-in for the open source 3D modeling software Blender [5], helps to generate morph targets by comparing the base model to a modified version and saving only the modified vertices.

As the deformation system libanimorph uses a special format to store the threedimensional model data, the tool PyExportMH provides the export of a model loaded 
in Blender to this format, including vertices, faces, vertex groups, materials and texture coordinates.

Another helper tool uses libanimorph and libavatar directly to calculate scales between real world and virtual construction measures.

In order to be able to analyze a range of results of a given morph target by applying different scalars, plottarget outputs a two-dimensional graph, containing the scalar value on the $\mathrm{x}$ - and the result of a measured hotspot on the $\mathrm{y}$-axis.

\subsection{Import and Export Capabilities}

The format to import data into the system is well-defined, but not the data itself. The import format is similar to Wavefront OBJ, but for various reasons split into several files. In principle, it is possible to use any three-dimensional geometric model as input data, independently of its topology. Using the present principle it is possible, for example, to build an automatic deformation system for a car model or a fantasy figure.

Currently supported export formats are Wavefront OBJ, Renderman RIB, and a proprietary format for the IntExMa project based on OBJ.

\subsection{Integration into Existing Software}

MakeHuman [3] is a multi-platform and open-source (GPL) software program whose purpose is to provide a versatile, professional and specialized application for parametrical modeling of three-dimensional humanoid characters. The MakeHuman application uses libanimorph as its rendering engine. The automatic deformation library libavatar is currently not used in MakeHuman. Currently, MakeHuman aims at the creation of nice-looking human avatars, not at the automatic reproduction of an individual real human.

In order to provide the users of the IntExMa system with a virtual counterpart, that looks as close to the real person as possible, the avatar generation system presented in this paper was integrated. As soon as new measurements are input by the salesperson the corresponding avatar is generated. Subsequently, a physically-based simulation clothes the virtual human with digitally represented garments. For more details about the IntExMa system please refer to [6].

\section{Results and Conclusions}

The quality of the results highly depends on the quality and quantity of the input data. The more detailed the base model is and the more morph targets respectively measuring sections are provided, the more precise the reproduction of the measures of the real person will be.

Fig. 3 shows two avatars resulting from the deformation process and dressed with a physically-based simulated shirt. The base model lies somewhere in between both figures. The avatars result from different morph targets that were specified for decreasing and for increasing measures (in relation to the base model). It can be stated, that the automatically generated avatars are visually appealing and suitable for the "virtual try-on" simulation. 

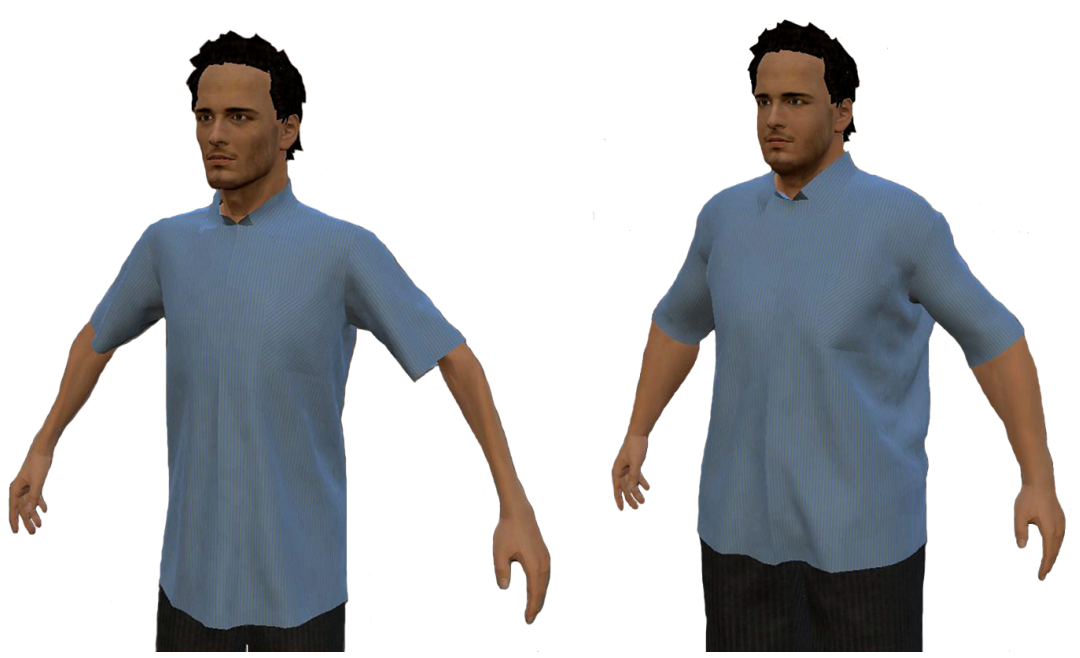

Fig. 3. Two avatars resulting from the same base model

At the same time, it must be considered that conventional length and girth body measures of a human do not fully specify the targeted outer characteristics of the respective body. The same set of measures may result from several real human bodies differing significantly in their appearance (muscle mass, posture etc.).

Nevertheless, it is not clear, how realistic the reproduction of the individual customer must be for the purpose at hand, the support of the buying decision and, accompanying, the general acceptance of the system concept.

Integrated into the overall IntExMa system, the efficiency of the presented approach for the "virtual try-on" of clothing and sales support will be evaluated within the scope of usability and acceptance tests with real customers and sales staff in a real made-to-measure tailor shop.

Acknowledgments. This research was carried out during the project IntExMa and financially supported by the German Federal Ministry of Education and Research.

\section{References}

1. Magnenat-Thalmann, N., Thalmann, D.: Handbook of Virtual Humans. John Wiley and Sons Ltd, New York (2004)

2. Lever, N.: Real-time 3D Character Animation with Visual C++, Focal Press (2002)

3. MakeHuman: (last retrieved 16/02/07) http://www.makehuman.org/

4. Humanoid animation (H-Anim): ( last retrieved 16/02/07) http://www.h-anim.org/

5. Blender: (last retrieved 16/02/07) http://www.blender.org/

6. Khakzar, K., Blum, R., Kohlhammer, J., Fuhrmann, A., Maier, A., Maier, A.: Interactive Product Visualization for an In-store Sales Support System for the Clothing Retail. In: Proceedings of HCII 2007, 12th International Conference on Human-Computer Interaction 2007 (to appear) 